\title{
A case of clinically silent giant right pheochromocytoma and review of literature
}

\author{
Changfu Li, MD; Yongsheng Chen, MD; Wentao Wang, MD; Lichen Teng, MD
}

Department of Urology, The Third Affiliated Hospital, Harbin Medical University, Harbin City, China

Cite as: Can Urol Assoc J 2012;6(6):E267-E269. http://dx.doi.org/10.5489/cuaj.11195

\section{Abstract}

Clinically silent giant cystic pheochromocytoma is a rare adrenal disease; complete resection is the only curative treatment. Due to the serious surgical risk, successful resection is very difficult. We report a case of a patient with giant cystic pheochromocytoma, which was successfully resected. There were no intraoperative and postoperative complications. We analyze and report our experience.

\section{Introduction}

Among the adrenal tumours, pheochromocytoma is special because of the catecholamines it produces. Catecholamines and their metabolite could result in a hypertension crisis during surgery. A large adrenal tumour $(>7 \mathrm{~cm})$ is generally defined as dysfunctional and malignant. More importantly, more attention is paid to clinically silent tumours that can be difficult to identify before surgery. Resection of the tumour should be the first treatment option. Successful resection is a great challenge for urologic surgeons. Due to a poorly exposed mass, potentially metastasis, and the high incidence of severe complications, a giant pheochromocytoma may be surgically unresectable. We report a patient with a clinically silent giant pheochromocytoma, which was successfully resected en bloc in May 2011. The patient had no complications during follow-up. In this paper, we report on our experience treating this patient.

\section{Case report}

A 56-year-old man, complaining of progressive weight loss and nausea for 3 months, was admitted to hospital in April 2011. He had no any history of paroxysmal hypertension and low back pain. On physical exam, there was no palpable abdominal mass. His blood pressure was $131 / 86 \mathrm{mmHg}$ and his pulse rate was $68 \mathrm{bpm}$. Laboratory exam found no abnormality. Abdominal computed tomography (CT) scan demonstrated a $12 \times 11 \times 11-\mathrm{cm}$ mass located between the

liver and right kidney, which occupied a large part of the right superior abdominal cavity. Contrast-enhanced CT scan showed a significantly enhanced solid area (Fig. 1). A magnetic resonance imaging scan revealed a11.1 $\times 10.4 \times 12.7-\mathrm{cm}$ mass, which dislocated the right kidney downward (Fig. 2 ). The mass displayed predominantly cystic and mild low signal on T1 weighted images; on the contrary, a mild high signal was found on T2 weighted images (Fig. 3). On the basis of this information and the patient's history, a malignant adrenal mass was suspected. Radical resection was the first option.

\section{Surgical technique}

The patient was placed in the supine position, with 20- to 30-degree flank position. The arc incision from the xiphoid process to right inferior costal margin was performed. After incising the flank peritoneum, we found a significant pulsatory feeling of the mass. These findings indicated that the mass had a sufficient blood circulation. With careful manipulation, we isolated the mass on the capsule.

During the process, blood spurted out from a small rupture on capsule of the mass. We mistakenly thought this was an arterial hemorrhage wanted to perform hemostasis by the way of transfixation; however, the manipulation resulted in increased blood loss because a hypertension crisis occurred. We had to stop any manipulation, until the blood pressure was controlled by the anesthetist. Finally, the inferior vena cava was exposed, a vessel with nearly $10 \mathrm{~mm}$ in diameter from the mass to the inferior vena cava was found, which was considered as the thickening right adrenal vein. The adrenal vein was ligated and dissected. As a result, hypotension suddenly presented. Transfusing blood and fluid treatment were immediately administered by an anesthetist. The mass was surgically excised en bloc. The cut surface of the mass appeared markedly cystic with a yellow gel. The area around the cyst consisted of kermesinus blood sinus. Blood pressure monitoring was not hypertensive or tachycardic.

The patient was discharged 8 days after surgery without antihypertensive treatment. Histopathologic and immunohistochemistric evaluation confirmed the diagnosis of pheo- 


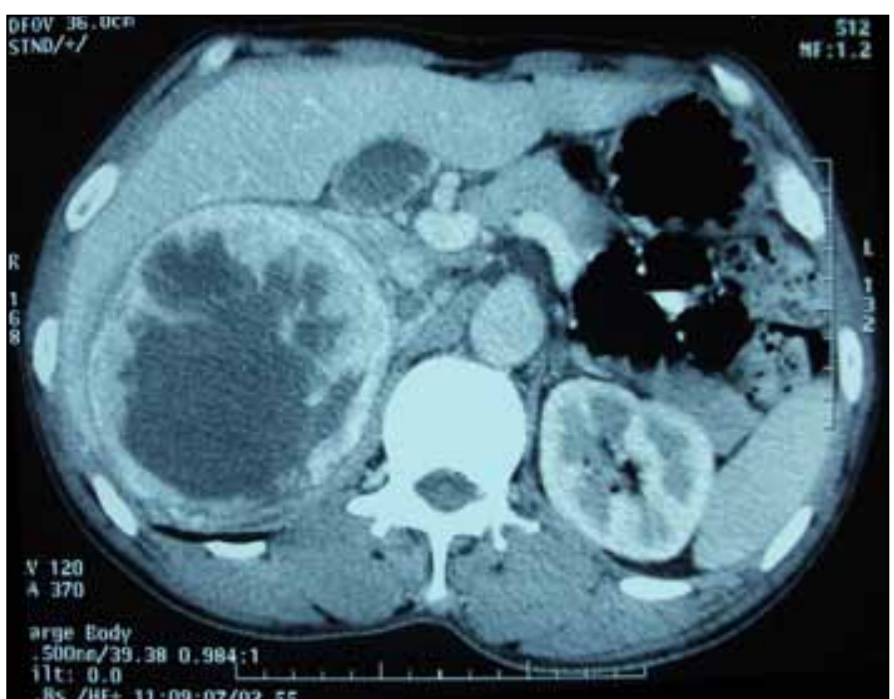

Fig 1. Post-contrast computed tomography axial plane shows the mass pressurizing liver forward and laterally. Outside area of the mass displays significant enhancement with uneven thickness. There is no evidence of local invasion.

chromocytoma. The immunohistochemistry found: $\mathrm{CgA}(+)$, inhibin(-), melanA(-), S100(-) and CK(-).

\section{Discussion}

Of all the adrenal pheochromocytoma, $20 \%$ to $30 \%$ of them are asymptomatic; they are called clinically silent pheochromocytoma. ${ }^{1,2}$ Generally, the neoplasm shows a larger tumour size at the time of diagnosis because of the delay to the first visit. On the basis of symptoms and signs, the patient should be defined as clinically silent pheochromocytoma. Different factors contribute to the diagnosis: (1) extensive necrosis cystic region at the centre of the mass may significantly decrease the number of cells producing catecholamine; (2) interstitial tissue without bioactivity may be the main ingredient of the neoplasm; considerable blood sinus around the cystic area is also demonstrated; (3) most of the catecholamines and metabolic product may be stored in the capsular mass, which infuses intravenously into the blood circulation when isolating the mass; as such, a hypertension crisis is possible.

The tumour size may be a variable. Generally, tumour sizes greater than $6 \mathrm{~cm}$ are malignant. However, in our case, the giant cystic mass was benign pheocrhomocytoma by histopathological evaluation. Although most urological surgeons thought the tumour was malignant in the preoperative discussion, so benign and malignant phaeochromocytoma cannot be distinguished on the basis of size. Goldstein and colleagues found that it was very difficult to predict the biological behaviour of pheochromocytoma, even if there were capsular invasion, vascular invasion and bizarre mitotic figures. A tumour can be benign or malignant on pathologic

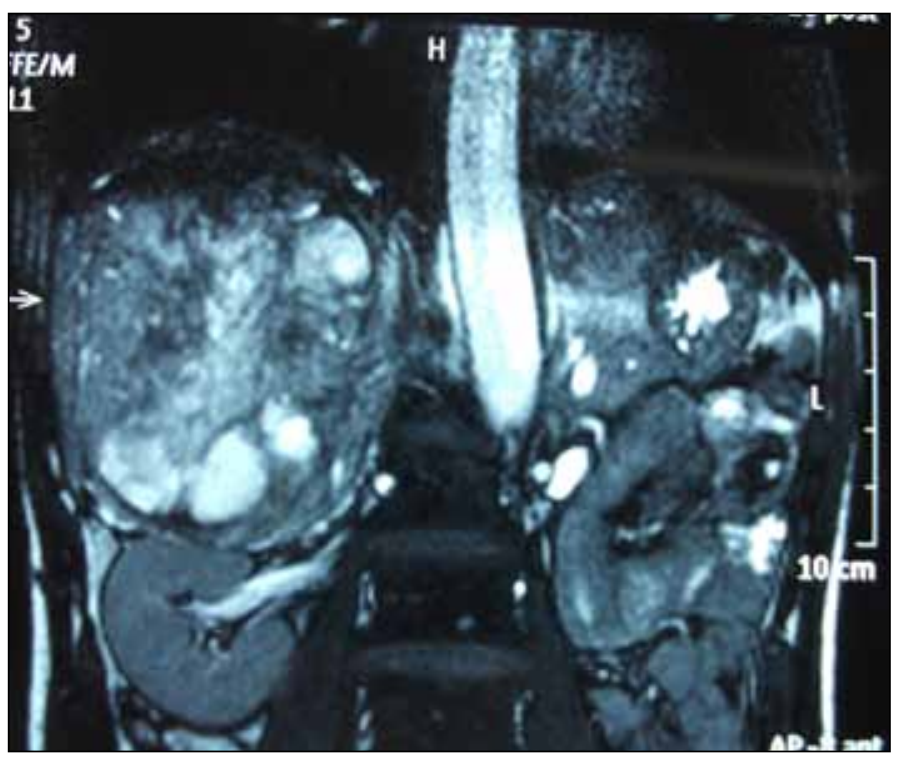

Fig 2. Magnetic resonance imaging coronal plane shows a giant well-defining round cystic right adrenal mass, dislocating from the right kidney downward.

evaluation. Apart from tumour size, a postoperative persistence of arterial hypertension was more frequent after the removal of malignant, no benign, tumours. ${ }^{3}$ In our case, blood pressure was normal postoperatively. The larger size cystic adrenal mass should not rule out the possibility of clinically silent pheochromocytoma; adequate preoperative preparation, including intravenous fluid expansion, is necessary. There are no guidelines to ensure adequate preoperative preparation in these clinically silent tumours. ${ }^{4}$

Once the adrenal neoplasm is diagnosed, resection is the only curative treatment. Although some reports have concluded that laparoscopic adrenalectomy is safe, effective, and minimally invasive, ${ }^{5-7}$ it is considered a gold standard in

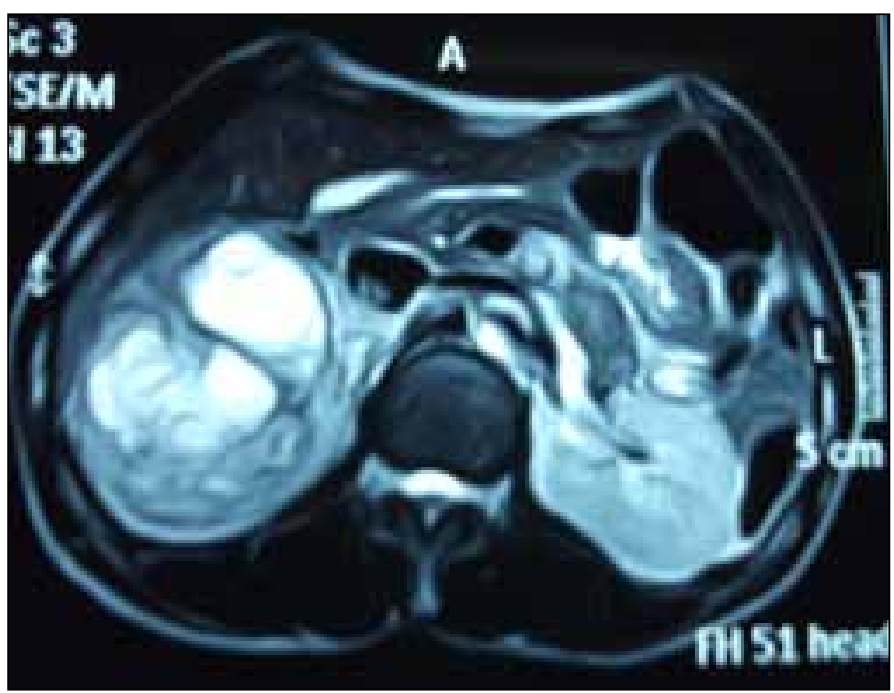

Fig 3. Magnetic resonance imaging axial plane demonstrates the mass with extensive cystic part. 
the surgical management of small benign adrenal tumours. In our case, considering the large size of the tumour $(>10 \mathrm{~cm})$, small surgical space and possible malignancy with local invasion, we performed traditional open surgery. Successful surgery is dependent on correctly exposing the capsule of the mass and preventing fracture of the capsule. In our case, the lesson suggested that controlling hemorrhage by transfixing the suture capsule was ineffective; quickly isolating and ligating the central adrenal vein was reasonable. On the other hand, according to our experience, the timing of the ligation of the central vein should be decided based on the function of the giant cystic pheochromocytoma. The central adrenal vein should be dissected and ligated earlier during the resection to reduce tumour size and control blood pressure. Moreover, blood loss would be decreased because most catecholamines would not drain into the blood, resulting in hypotenstion. Wang Dong and colleagues reported that selective arterial embolization before surgery can significantly decrease blood loss. ${ }^{8}$ However, due to the extensive abnormal vascularization with multiple arterio-venous communication, the authors found it difficult to complete arterial embolization. Additionally, with resection of the giant adrenal pheochromocytoma on the left side, there was more surgical risk for the resection on the right side. ${ }^{9}$

Competing interests: None declared.
This paper has been peer-reviewed.

\section{References}

1. Andreoni C, Krebs RK, Bruna PC, et al. Cystic phaeochromocythoma is a distintive subgroup with special clinic,imaging and histological features that might mislead the diagnosis. BJU Int 2008;101:345-50. http://dx.doi.org/10.1111/j.1464-410X.2007.07370.x

2. Antedomenico E, Wascher RA. A case of mistaken identity: giant cystic pheocromocytoma. Curr Surg 2005;62:193-8. http://dx.doi.org/10.1016/i.cursur.2004.08.015

3. Park J, Song C, Park M, et al. Predictive characteristics of malignant pheochromocytoma. Korean J Urol 2011;52:241-6. Epub 2011 Apr 22. http://dx.doi.org/10.4111/kju.2011.52.4.241

4. Shen SJ, Cheng HM, Chiu AW, et al. Perioperative Hypertensive Crisis in Clinically Silent Pheochromocytomas: Report of Four Cases. Chang Gung Med J 2005;28:44-50.

5. Brix D, Allolio B, Fenske W, et al. Laparoscopic versus open adrenalectomy for adrenocortical carcinoma: surgical and oncologic outcome in 152 patients. Eur Urol 2010;58:609-15. Epub 2010 Jun 22. http:// dx.doi.org/10.1016/i.eururo.2010.06.024

6. Wang HS, Li CC, Chou YH, et al. Comparison of laparoscopic adrenalectomy with open surgery for adrenal tumors. Kaohsiung J Med Sci 2009;25:438-44. http://dx.doi.org/10.1016/S1607-551X(09)70539-X

7. Tiberio GA, Baiocchi GL, Arru L, et al. Prospective randomized comparison of laparoscopic versus open adrenalectomy for sporadic pheochromocytoma. Surg Endosc 2008;22:1435-9. Epub 2008 Apr 9. http:// dx.doi.org/10.1007/s00464-008-9904-1

8. Wang Dong, Li Hanzhong, Zhang Yushi, et al. Selective arterial emblization in giant pheochromocytoma. Zhong Hua Mi Niao Wai Ke Za Zhi 2011;32:299-302.

9. Tatsugami K, Eto M, Hamaguchi $M$, et al. What affects the results of a laparoscopic adrenalectomy for pheochromocytoma? Evaluation with respect to intraoperative blood pressure and state of tumor. Endourol 2009;23:101-5. http://dx.doi.org/10.1089/end.2008.0279

Correspondence: Dr. Lichen Teng, Department of Urology, The Third Affiliated Hospital, Harbin Medical University, Harbin City, China 150081; tenglichen@2008.sina.com 